\title{
Axial current in rotating and accelerating medium
}

\author{
George Y. Prokhorov, ${ }^{1, *}$ Oleg V. Teryaev, ${ }^{1,2,3, \dagger}$ and Valentin I. Zakharov ${ }^{2,4, \hbar}$ \\ ${ }^{1}$ Joint Institute for Nuclear Research, 141980 Dubna, Russia \\ ${ }^{2}$ Institute of Theoretical and Experimental Physics, B. Cheremushkinskaya 25, 117218 Moscow, Russia \\ ${ }^{3}$ National Research Nuclear University MEPhI (Moscow Engineering Physics Institute), \\ Kashirskoe Shosse 31, 115409 Moscow, Russia \\ ${ }^{4}$ School of Biomedicine, Far Eastern Federal University, 690950 Vladivostok, Russia
}

(Received 15 June 2018; published 26 October 2018)

\begin{abstract}
Statistical average of the axial current is evaluated on the basis of the covariant Wigner function. In the resulting formula, chemical potential $\mu$, angular velocity $\Omega$ and acceleration $|\boldsymbol{a}|$ enter in combination $\mu \pm(\Omega \pm i|\boldsymbol{a}|) / 2$. The limiting cases of zero mass and zero temperature are investigated in detail. In the zero-mass limit, the axial current is described by a smooth function only at temperatures higher than the Unruh temperature. At zero temperature, the axial current, as a function of the angular velocity and chemical potential, vanishes in a two-dimensional plane region.
\end{abstract}

DOI: 10.1103/PhysRevD.98.071901

\section{INTRODUCTION}

Recently many remarkable effects related to the properties of relativistic fluids have been discovered at the theoretical level. The nature of these effects, on one hand, is associated with fundamental properties of matter, and, on the other hand, they are expected to be observable experimentally. Two best known examples of this kind are the chiral magnetic (CME) and the chiral vortical effect (CVE) manifested in electromagnetic and axial currents, respectively. For detailed discussion of the effects we refer the reader to the rich existing literature, see in particular [1-14].

In $[11,15]$ the mean value of the axial current was calculated on the basis of the ansatz for the covariant Wigner function proposed in [16]. The resulting formula reduces to the standard formula for the CVE in the approximation linear in vorticity.

Moreover, as is emphasized in [15], the entire series of expansion in the thermal vorticity can be summed up. The result contains information on corrections to the standard CVE. Some of these higher order terms have been derived earlier within other approaches [1,2]. Here we demonstrate that the formula obtained can be greatly simplified and reduced to a form in which the angular velocity and acceleration enter as a real and imaginary chemical potentials, respectively. Due to this, the axial current acquires

\footnotetext{
*prokhorov@theor.jinr.ru

teryaev@theor.jinr.ru

vzakharov@itep.ru
}

Published by the American Physical Society under the terms of the Creative Commons Attribution 4.0 International license. Further distribution of this work must maintain attribution to the author(s) and the published article's title, journal citation, and DOI. Funded by SCOAP. features inherent in theories with imaginary chemical potential, in particular, periodic discontinuities or cusps appear at a temperature below the Unruh temperature. According to [17] for linearly accelerated systems, the Unruh temperature is the lowest possible temperature. Our observation on existence of discontinuities in the behavior of the axial current at temperatures below the Unruh temperature supports this conclusion. We also investigated how this boundary varies with the rotation. Note that existence of a boundary temperature proportional to the Unruh temperature was also derived in [18] starting from the condition of positivity of energy density.

Despite the fact that the results obtained relate to the theories of weakly interacting fermions, we give arguments in favor of their validity, even in the case of strong coupling, at least on a qualitative level.

Evaluation of the axial current might have important phenomenological implications. Indeed, the appearance of a significant baryon polarization in heavy ion collisions can be one of most important experimental signatures of the CVE. In particular, papers in Refs. [19-22], relate the polarization of baryons to an anomalous axial charge of quarks. On the other hand, the polarization effects can be investigated within the framework of the relativistic hydrodynamics of baryons [23-25], based on the Wigner function introduced in [16], from which the CVE can also be derived. Note that the carriers of the axial charge differ in the two approaches. This situation served as a motivation for us to study the effects in the axial current $[15,16]$, connected with a finite mass of particles. An interesting phenomenon, which we find in this case, is the existence of a planar two-dimensional domain in the coordinates $\Omega, \mu$, where the axial current vanishes. Qualitatively, such a picture is associated with the above-mentioned observation 
that the angular velocity plays the role of a new chemical potential.

The system of units $\hbar=c=k=1$ is used.

\section{ANALYSIS OF THE EFFECTS OF MOTION OF THE MEDIUM ON THE BASIS OF THE WIGNER FUNCTION}

As is known, kinetic properties of a medium can be derived from the quantum field theory using the Wigner function, see, e.g., [26]. In the Ref. [16] an ansatz for the Wigner function was proposed to describe media with fermionic constituents in the state of a local thermodynamic equilibrium. Moreover, it was checked that the ansatz reproduces correctly some known limiting cases. Based on this ansatz, the effects associated with thermal vorticity were investigated in various physical quantities $[11,15,16,18]$. In particular, in $[11,15]$, the axial current was first calculated, while an exact formula within the framework of this formalism was obtained in [15].

The Wigner function in [16] is expressed in terms of the distribution function $X(x, p)$, which has the form of a modified Fermi-Dirac distribution

$X(x, p)=\left(\exp \left[\beta_{\mu} p^{\mu}-\zeta\right] \exp \left[-\frac{1}{2} \varpi_{\mu \nu} \Sigma^{\mu \nu}\right]+I\right)^{-1}$,

where $\zeta=\frac{\mu}{T}, \varpi_{\mu \nu}$ is the thermal vorticity tensor, and $\Sigma_{\mu \nu}=\frac{i}{4}\left[\gamma_{\mu}, \gamma_{\nu}\right]$. Mean values of various physical quantities can be found by integrating the trace of the operator of the quantity considered with the function $X(x, p)$ over the momentum space. Thus, for the axial current we have the following formula [16]

$\left\langle j_{\mu}^{5}\right\rangle=-\frac{1}{16 \pi^{3}} \epsilon_{\mu \alpha \nu \beta} \int \frac{d^{3} p}{\varepsilon} p^{\alpha}\left\{\operatorname{tr}\left(X \Sigma^{\nu \beta}\right)-\operatorname{tr}\left(\bar{X} \Sigma^{\nu \beta}\right)\right\}$,

where $\langle\cdot\rangle$ denotes statistical averaging with normal ordering, $\bar{X}$ describes the contribution of antiparticles and differs from (2.1) in sign of $\zeta$ and $\varpi$. The matrix traces in (2.2) were exactly found in [15] in formula (4.3)

$$
\begin{aligned}
\operatorname{tr}\left(X \Sigma^{\nu \beta}\right)= & \left\{\left(\exp \left[(\beta p)-\zeta-\frac{g_{\omega}}{2 T}+i \frac{g_{a}}{2 T}\right]+1\right)^{-1}\right. \\
& \left.-\left(\exp \left[(\beta p)-\zeta+\frac{g_{\omega}}{2 T}-i \frac{g_{a}}{2 T}\right]+1\right)^{-1}\right\} \\
& \times \frac{T}{2\left(g_{\omega}-i g_{a}\right)}\left[\varpi^{\nu \beta}-i \operatorname{sgn}\left(\varpi_{\mu \alpha} \tilde{\varpi}^{\mu \alpha}\right) \tilde{\varpi}^{\nu \beta}\right]+\text { c.c., }
\end{aligned}
$$

where $\tilde{\varpi}^{\nu \beta}$ is the tensor dual to $\varpi^{\nu \beta}$, while $g_{\omega}$ and $g_{a}$ are scalar quantities that depend on acceleration $a^{\mu}=u^{\nu} \partial_{\nu} u^{\mu}$ and vorticity $\omega_{\mu}=\frac{1}{2} \epsilon_{\mu \nu \alpha \beta} u^{\nu} \partial^{\alpha} u^{\beta}$ $g_{\omega}=\frac{1}{\sqrt{2}}\left(\sqrt{\left(a^{2}-\omega^{2}\right)^{2}+4(\omega a)^{2}}+a^{2}-\omega^{2}\right)^{1 / 2}$,
$g_{a}=\frac{1}{\sqrt{2}}\left(\sqrt{\left(a^{2}-\omega^{2}\right)^{2}+4(\omega a)^{2}}-a^{2}+\omega^{2}\right)^{1 / 2}$,

where $a^{2}=a_{\mu} a^{\mu}, \quad \omega^{2}=\omega_{\mu} \omega^{\mu} \quad$ and $\quad(\omega a)=\omega_{\mu} a^{\mu}$. Substituting (2.3) into (2.2), we obtain

$$
\begin{aligned}
\left\langle j_{\mu}^{5}\right\rangle= & \frac{\omega_{\mu}+i \operatorname{sgn}(\omega a) a_{\mu}}{2\left(g_{\omega}-i g_{a}\right)} \int \frac{d^{3} p}{(2 \pi)^{3}} \\
& \times\left\{n_{F}\left(E_{p}-\mu-g_{\omega} / 2+i g_{a} / 2\right)\right. \\
& -n_{F}\left(E_{p}-\mu+g_{\omega} / 2 i g_{a} / 2\right) \\
& +n_{F}\left(E_{p}+\mu-g_{\omega} / 2+i g_{a} / 2\right) \\
& \left.-n_{F}\left(E_{p}+\mu+g_{\omega} / 2-i g_{a} / 2\right)\right\}+ \text { c.c., }
\end{aligned}
$$

which is another form of Eq. (4.6) from [15]. Here $n_{F}(E)=$ $\left(e^{E / T}+1\right)^{-1}$ is the Fermi-Dirac distribution.

It is useful to consider a particular case by going into the comoving reference system, in which $a^{\mu}=(0, \boldsymbol{a})$ and $\omega^{\mu}=(0, \boldsymbol{\Omega})$, and assuming that $\boldsymbol{\Omega} \| \boldsymbol{a}$, that is, the acceleration directed along the rotation axis. Then $a^{2}=-|\boldsymbol{a}|^{2}$, $\omega^{2}=-\Omega^{2}$ and $(\omega a)^{2}=\Omega^{2}|\boldsymbol{a}|^{2}$, which leads to $g_{\omega}=\Omega$, $g_{a}=|\boldsymbol{a}|$. Then (2.5) leads to

$$
\begin{aligned}
\left\langle\boldsymbol{j}^{5}\right\rangle= & \frac{1}{2} \int \frac{d^{3} p}{(2 \pi)^{3}}\left\{n_{F}\left(E_{p}-\mu-\frac{\Omega}{2}+i \frac{|\boldsymbol{a}|}{2}\right)\right. \\
& -n_{F}\left(E_{p}-\mu+\frac{\Omega}{2}+i \frac{|\boldsymbol{a}|}{2}\right) \\
& +n_{F}\left(E_{p}+\mu-\frac{\Omega}{2}+i \frac{|\boldsymbol{a}|}{2}\right) \\
& \left.-n_{F}\left(E_{p}+\mu+\frac{\Omega}{2}+i \frac{|\boldsymbol{a}|}{2}\right)+\text { c.c. }\right\} \boldsymbol{e}_{\Omega},
\end{aligned}
$$

where $\boldsymbol{e}_{\Omega}=\frac{\Omega}{\Omega}$ is the unit vector in the direction of the angular velocity.

Equation (2.6) demonstrates that $\Omega$ and $|\boldsymbol{a}|$ come in a certain combination with the chemical potential. Thus, the effect of rotation and acceleration reduces to a modification of the chemical potential and introduction of a kind of an imaginary chemical potential. This conclusion is worthy of further discussion, but we note that the possibility of considering angular velocity as a chemical potential has already been discussed in the literature [27]. It is interesting to note that, according to Eq. (2.6), the axial current turns out to be formally a periodic function of acceleration $|\boldsymbol{a}|$, that is, it does not change with $\frac{|\boldsymbol{a}|}{2} \rightarrow \frac{|\boldsymbol{a}|}{2}+2 \pi T n$, $n=0, \pm 1, \pm 2 \ldots$ Apparently, this behavior is similar to the Roberge-Weiss periodicity existing in theories with an imaginary chemical potential, where the partition function also turns out to be periodic with respect to the imaginary 
chemical potential $[28,29]$ (without obvious relation of the period to the phase transition, as boost appears instead of particle number). It should be also noted that for $|\boldsymbol{a}|>2 \pi T$ we go beyond the allowed acceleration values, as follows from $[17,18]$, as well as the analysis given below.

In the limiting case of massless fermions, $m=0$, the integrals in (2.5) can be found analytically and expressed in terms of polylogarithms in the same way as was done in [15]. Using the following property of the polylogarithms [30]

$$
\begin{aligned}
\mathrm{Li}_{3}\left(-e^{a+i b}\right)-\mathrm{Li}_{3}\left(-e^{-a-i b}\right) & -\frac{1}{6}\left\{a+2 \pi i\left[\frac{b}{2 \pi}-\left\lfloor\frac{b}{2 \pi}+\frac{\operatorname{sgn}(b)}{2}\right]\right]\right\}^{3} \\
= & -\frac{\pi^{2}}{6}\left\{a+2 \pi i\left[\frac{b}{2 \pi}-\left\lfloor\frac{b}{2 \pi}+\frac{\operatorname{sgn}(b)}{2}\right]\right]\right\},
\end{aligned}
$$

we obtain

$$
\begin{aligned}
\left\langle j_{\mu}^{5}\right\rangle= & \left(\frac{1}{6}\left[T^{2}+\frac{a^{2}-\omega^{2}}{4 \pi^{2}}\right]+\frac{\mu^{2}}{2 \pi^{2}}\right) \omega_{\mu}+\frac{1}{12 \pi^{2}}(\omega a) a_{\mu}+\omega_{\mu}\left[-\frac{4 \pi T g_{a}}{g_{a}^{2}+g_{\omega}^{2}}\left(\frac{T^{2}}{6}+\frac{\mu^{2}}{2 \pi^{2}}-\frac{g_{a}^{2}}{8 \pi^{2}}-\frac{g_{\omega}^{2}}{8 \pi^{2}}\right)\left\lfloor\frac{g_{a}}{4 \pi T}+\frac{1}{2}\right\rfloor\right. \\
& \left.-2 T^{2}\left\lfloor\frac{g_{a}}{4 \pi T}+\frac{1}{2}\right\rfloor^{2}+\frac{8 \pi T^{3} g_{a}}{3\left(g_{a}^{2}+g_{\omega}^{2}\right)}\left\lfloor\frac{g_{a}}{4 \pi T}+\frac{1}{2}\right\rfloor^{3}\right]+a_{\mu} \operatorname{sgn}(\omega a)\left[-\frac{4 \pi T g_{\omega}}{g_{a}^{2}+g_{\omega}^{2}}\left(\frac{T^{2}}{6}+\frac{\mu^{2}}{2 \pi^{2}}+\frac{g_{a}^{2}}{8 \pi^{2}}+\frac{g_{\omega}^{2}}{8 \pi^{2}}\right)\left\lfloor\frac{g_{a}}{4 \pi T}+\frac{1}{2}\right\rfloor\right. \\
& \left.+\frac{8 \pi T^{3} g_{\omega}}{3\left(g_{a}^{2}+g_{\omega}^{2}\right)}\left\lfloor\frac{g_{a}}{4 \pi T}+\frac{1}{2}\right\rfloor^{3}\right],
\end{aligned}
$$

where $\lfloor\cdot\rfloor$ is the integer part. Note that in [15] the particular case $\left|\frac{b}{2 \pi}+\frac{\operatorname{sgn}(b)}{2}\right|<1$ was considered under which formula (2.7) leads to the Eq. (4.9) from [15], which means that resulting formula Eq. (4.11) from [15] corresponds to the case $T>\frac{g_{a}}{2 \pi}$. Due to contributions from $\left\lfloor\frac{g_{a}}{4 \pi T}+\frac{1}{2}\right\rfloor$ for $T<\tilde{T}_{U}$, where $\tilde{T}_{U}$ is

$$
\tilde{T}_{U}=\frac{g_{a}}{2 \pi},
$$

the formula (2.8) has discontinuities or cusps. More precisely, according to (2.8) the axial current (its projections, modulus and derivatives) have discontinuities (or cusps) at $\frac{g_{a}}{2}=(2 n+1) \pi T, n=0,1,2 \ldots$ These discontinuities look like Roberge-Weiss phase transitions found in theories with imaginary chemical potential, which also repeat periodically $[28,29]$. For $T>\tilde{T}_{U}$ the formula (2.8) takes the form of Eq. (4.11) from [15], derived in approximation $T>\tilde{T}_{U}$

$$
\left\langle j_{\mu}^{5}\right\rangle=\left(\frac{1}{6}\left[T^{2}+\frac{a^{2}-\omega^{2}}{4 \pi^{2}}\right]+\frac{\mu^{2}}{2 \pi^{2}}\right) \omega_{\mu}+\frac{1}{12 \pi^{2}}(\omega a) a_{\mu} .
$$

It is interesting to note that in the case of $\boldsymbol{\Omega} \| \boldsymbol{a}$ or $\boldsymbol{\Omega}=0$ the condition $T>\tilde{T}_{U}$ results in $T>\frac{|a|}{2 \pi}$, that is, the temperature is to be greater than the Unruh temperature $T_{U}=\frac{|a|}{2 \pi}$. The appearance of the Unruh temperature in Eq. (2.8) is a direct consequence of the fact that in (2.5) and (2.6) the acceleration enters as an imaginary chemical potential. If both acceleration and angular velocity are nonzero and directed arbitrarily, the boundary temperature is generalized to $T_{U} \rightarrow \tilde{T}_{U}(\Omega,|\boldsymbol{a}|, \theta)$, where $\theta$ is the angle between $\boldsymbol{a}$ and $\boldsymbol{\Omega}$ in the comoving reference system.

According to [17], the Unruh temperature is the minimum temperature that an accelerated medium can have. Apparently, this fact is the reason why the behavior of the axial current in Eq. (2.8) changes qualitatively at $T<\tilde{T}_{U}$. A similar result on the existence of a boundary temperature proportional to the Unruh temperature on the basis of the same Wigner function [16] was recently obtained in [18] by considering the energy-momentum tensor and the condition of positivity of the energy density. We note, however, that in [18] the boundary temperature is twice that of the Unruh temperature.

In [17] the boundary of possible values of acceleration and temperature for medium without rotation has the form of a straight line $T_{U}=\frac{|\boldsymbol{a}|}{2 \pi}$ in the plane $|\boldsymbol{a}|, T$ : this boundary is shown on the left side of the Fig. 1 with a blue dot-dashed line. The region below this line, following [17], is forbidden. Using the condition that the axial current remains a continuous smooth function of temperature and acceleration, as a criterion for determining possible values, we can study how the boundary of allowed values moves as the angular velocity modulus $\Omega$ and angle between acceleration and angular velocity $\theta$ change. This condition is equivalent to the requirement $T>\tilde{T}_{U}$, Eq. (2.9), as was shown before. In this case, at $\boldsymbol{\Omega} \| \boldsymbol{a}$, the result [17] is reproduced and the boundary is given by the line $T_{U}=\frac{|\boldsymbol{a}|}{2 \pi}$ corresponding to the blue dot-dashed line on the left side of Fig. 1. For perpendicular $\boldsymbol{\Omega} \perp \boldsymbol{a}$, the boundary shifts and becomes according to Eq. (2.9)

$$
\tilde{T}_{U}=\frac{\sqrt{|\boldsymbol{a}|^{2}-\Omega^{2}}}{2 \pi}
$$



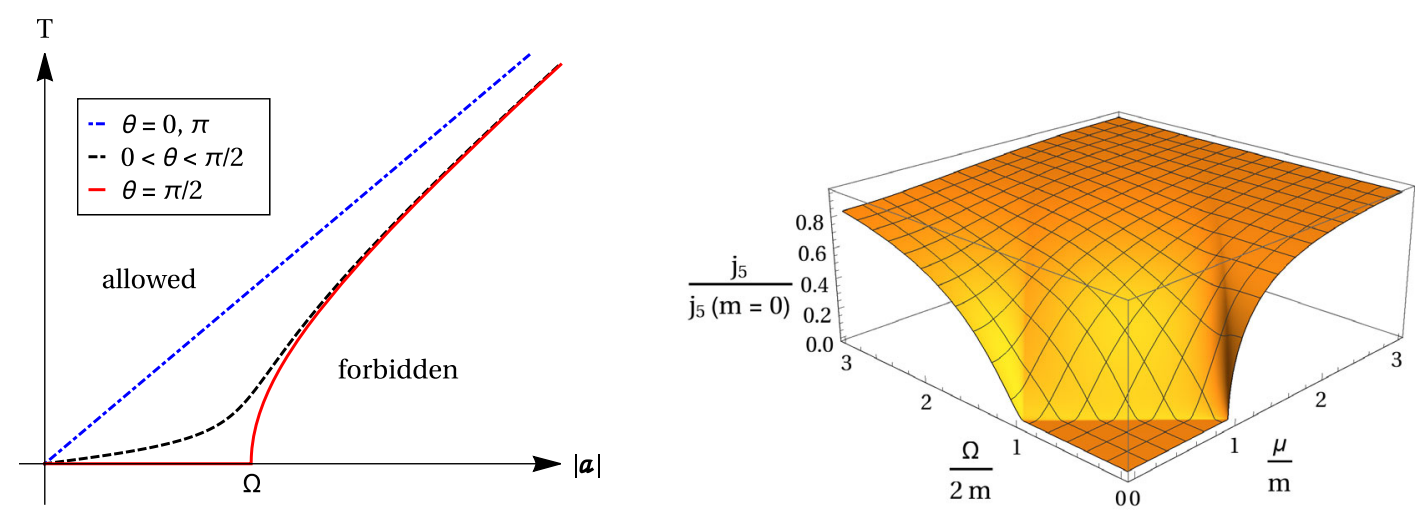

FIG. 1. Left: curves corresponding to the appearance of instability in axial current on the plane $|\boldsymbol{a}|, T$. The dot-dashed blue line corresponds to the angles $\theta=0, \pi$ (also $\Omega=0$ ) between the acceleration and the angular velocity, solid red-to the angle $\theta=\pi / 2$, the dashed black - to the angles $0<\theta<\pi / 2$ (and also $\pi / 2<\theta<\pi$ ). The area below these curves, presumably, is the forbidden region of the values of acceleration and temperature. Right: axial current (3.1), as a function of the chemical potential and angular velocity at zero temperature. The value of $j_{5}=\left|\left\langle\boldsymbol{j}_{5}\right\rangle\right|$ is normalized to its value (2.10) at zero mass.

for $|\boldsymbol{a}|>\Omega$ and 0 for $|\boldsymbol{a}|<\Omega$, which corresponds to the red solid line on the left side of Fig. 1. For intermediate values of the angle $\theta$, the boundary is between the boundaries for $\boldsymbol{\Omega} \perp \boldsymbol{a}$ and $\boldsymbol{\Omega} \| \boldsymbol{a}$, which corresponds to the black dashed line on the Fig. 1.

Note that (2.10) in the first order in $\omega^{\mu}$ leads to the standard formula for CVE $[11,15]$, while $\left(-\frac{\omega^{2}}{24 \pi^{2}}\right) \omega_{\mu}$ is consistent with the results of $[1,2]$ (see also [31] for recent progress in the geometric approach, developed in [1]).

\section{EFFECTS OF FINITE MASS}

There exist various approaches to calculating the polarization of baryons in heavy ion collisions. In particular, in the [19-22] the axial charge of quarks, acquired by them due to the CVE, is considered, and this charge is associated with the polarization of baryons. On the other hand, in [23-25], the polarization is calculated on the basis of the Wigner function for a medium consisting of baryons, assuming equilibrium of the spin degrees of freedom.

Note that the CVE, which is essential for calculating the polarization in [19-22], arises in the approach of Refs. [23-25] as well. However, in [19-22], quarks are considered as carriers of the axial charge, while in [23-25] they are baryons, that is, particles with different masses. In view of this, it is useful to consider the effects of a finite mass in an axial current.

The most characteristic features in the behavior of the axial current arise at $T=0$. For simplicity, we also assume that $a_{\mu}=0$. Going into the comoving reference frame, we obtain $g_{a}=0$ and $g_{\omega}=\Omega$ in (2.4). The integrals in (2.6) can be evaluated analytically, and we get a simple formula

$$
\begin{aligned}
\left\langle j^{5}\right\rangle= & \frac{1}{6 \pi^{2}}\left\{\theta\left(\mu+\frac{\Omega}{2}-m\right)\left[\left(\mu+\frac{\Omega}{2}\right)^{2}-m^{2}\right]^{3 / 2}\right. \\
& -\theta\left(\mu-\frac{\Omega}{2}-m\right)\left[\left(\mu-\frac{\Omega}{2}\right)^{2}-m^{2}\right]^{3 / 2} \\
& +\theta\left(-\mu+\frac{\Omega}{2}-m\right)\left[\left(\mu-\frac{\Omega}{2}\right)^{2}-m^{2}\right] 3 / 2 \\
& \left.-\theta\left(-\mu-\frac{\Omega}{2}-m\right)\left[\left(\mu+\frac{\Omega}{2}\right)^{2}-m^{2}\right]^{3 / 2}\right\} \boldsymbol{e}_{\Omega},
\end{aligned}
$$

where $\theta$ is the Heaviside function. From (3.1) it follows, in particular, that for $\Omega<2(m-|\mu|)$ the axial current is zero. This is in accord with the absence of chemical-potential effect if $\mu$ is smaller than the corresponding physical masses. Moreover, we find out that in case of a rotating medium, this is true for the "effective" chemical potential incorporating the angular velocity.

The behavior of $j_{5}=\left|\left\langle\boldsymbol{j}_{\mathbf{5}}\right\rangle\right|$, see Eq. (3.1), as a function of $\Omega$ and $\mu$ is shown in Fig. 1 on the right. For $\Omega \gg m$ and $\mu \gg m$ the axial current asymptotically tends to its value at zero mass Eq. (2.10), $j_{5}(m=0)=\left(\frac{\Omega^{2}}{24 \pi^{2}}+\frac{\mu^{2}}{2 \pi^{2}}\right) \Omega$, as it should be. In general, due to the effects associated with the mass, $j_{5}$ in the massive case is always smaller than in the massless limit, as can be seen from Fig. 1 on the right.

\section{BEYOND THE APPROXIMATION MADE: DISCUSSION}

Equations (2.6) and (2.8) exhibit features which are challenging to explain theoretically on general grounds. Moreover, the results are obtained in a certain approximation which utilizes exclusively various moments from the 
Fermi distribution valid for noninteracting Fermi gas. And a crucial question is whether the results survive with account of interaction. At this section, we comments briefly on these questions, emphasizing the possibility of validity of the results, at least qualitatively, beyond the approximation of noninteracting fermions. A full answer would require efforts which go beyond the scope of the present paper.

The first question is, how the imaginary acceleration, $i|\boldsymbol{a}|$ arises in Eq. (2.6) at all. Indeed, originally, see [17] and references therein, the acceleration $|\boldsymbol{a}|$ enters the density operator $\hat{\rho}$ as a real number. For example, in the absence of rotation,

$$
\hat{\rho}=(1 / Z) \exp \left(-\hat{H} / T_{0}+|\boldsymbol{a}|_{0} \hat{K}_{z} / T_{0}\right),
$$

where $\hat{H}$ is the Hamiltonian and $\hat{K}_{z}$ is the generator of a Lorentz boost along the $z$-axis ( $z$-axis is directed along acceleration, for the connection of $T_{0},|\boldsymbol{a}|_{0}$ with $T,|\boldsymbol{a}|$ look [17]). In this sense, the Eq. (4.1) looks as a straightforward generalization of the standard textbook equilibrium density operator.

However, when applied to a spinor field in an irreducible representations the boost operator results in a complex number, see, e.g., [32]. Indeed, the angular momentum $\hat{J}$ and boost generator $\hat{K}$ are combined into

$$
\hat{N}=\hat{J}+i \hat{K}, \quad \hat{N}^{\dagger}=\hat{J}-i \hat{K},
$$

where the corresponding eigenvalues $N \neq 0, N^{\dagger}=0$ for left-handed spinors and $N^{\dagger} \neq 0, N=0$ for right-handed spinors. This leads to opposite signs of the (imaginary) acceleration for left- and right-handed fermions. Thus, emergence of the imaginary acceleration in Eq. (2.6) is rooted in general rules of constructing Lorentz-invariant Lagrangian of fermionic fields and, apparently, is not specific for the approximation of free fields.

Turn now to the question of validity of the results obtained beyond the approximation of noninteracting fermions. Consider first the axial current $\left\langle j^{5}\right\rangle$ in Eq. (2.10) for $a_{\mu}=0$, evaluated in the scheme considered in the leading approximation $[11,15]$ :

$$
\left\langle j^{5}\right\rangle=\left(\frac{\mu^{2}}{2 \pi^{2}}+\frac{T^{2}}{6}+\frac{\Omega^{2}}{24 \pi^{2}}\right) \boldsymbol{\Omega} .
$$

In fact, the result (4.3) goes back to calculations of Vilenkin [1] and refers to the current of noninteracting relativistic fermions in a rotating frame at finite chemical potential $\mu$ and temperature $T$. On the other hand, as it was clarified rather recently (see for references, e.g., the volume [4]), Eq. (4.3) is valid as well in case of strongly interacting media, in the hydrodynamic approximation. This "miraculous" coincidence of the results in the noninteracting and strong-coupling cases can be demonstrated in two steps (for a recent review see [33]). First, moments from the Fermi distribution turn to capture the effect of the chiral anomaly and, second, the anomaly, being topological in nature, does not receive contributions from higher loops.

The derivation is easiest to outline in case of the $\mu^{2}$ term in Eq. (4.3), see, e.g., [34]. Indeed, it is straightforward to argue, see, e.g., [9], that in the hydrodynamic approximation the standard electromagnetic potential $A_{\alpha}$ in vertices is replaced by the combination:

$$
e A_{\alpha} \rightarrow e A_{\alpha}+\mu u_{\alpha} .
$$

As a result of this substitution the $\mu^{2}$ term in Eq. (4.3) is generated as an extension of the standard triangle anomalous graph. Generalized Adler-Bardeen theorem guarantees the absence of higher-order corrections.

An independent, anomaly-related evaluation of the $T^{2}-$ and $\Omega^{2}-$ terms in Eq. (4.3) requires consideration of noninertial frames, or nontrivial gravitational fields and is much more involved. The point is that consideration of motion with permanent acceleration introduces space-time with a boundary, or horizon. The crucial observation [35] is that while the gravitational chiral anomaly [36] is negligible in the bulk, it turns to be crucial on the edge, or on the horizon. Effectively, the anomaly becomes twodimensional and pumps chiral fermions into the bulk. Inside the bulk the flow of particles is conserved. This mechanism represents an alternative (or dual) description of the Hawking radiation of the chiral fermions. If one starts with field theory at $T=0$ the axial current, supported by the gravitational chiral anomaly coincides with (4.3) provided that

$$
T^{2} \rightarrow T_{H}^{2},
$$

where $T_{H}$ is the temperature of the Hawking radiation in case of the rotating black hole.

In this sense, the expression (4.3) which is formally derived in the no-interaction approximation [1] reproduces, upon the substitution (4.4) the Hawking radiation of chiral fermions which is a general dynamic phenomenon manifested in interacting case as well.

Moreover, the phenomenological analysis of Sec. II indicates clearly instability of the axial current if the temperature of the medium $T$ is chosen below the Unruh, or Hawking temperature. In the approximation considered, the instability is entirely due to the imaginary acceleration. On the other hand, the instability is nothing else but another manifestation of the Unruh, or Hawking radiation. This chain of arguments supports phenomenological introduction of the imaginary acceleration in case of chiral fermions.

In any case the term with $\mu^{2}$ remains stable, and since the effects observed look like an addition to the chemical potential (like emergence of a covariant 
derivative), it is likely that they will also remain in the theory with interaction.

The coefficient $1 / 2$ in front of $\Omega$ in this "modified chemical potential", $\mu+\Omega / 2$ can be interpreted as a consequence of the equivalence principle according to which spin and angular momentum precess with the same angular velocity [37]. In other words, the spin precession is twice slower than in the case of magnetic field. This factor of $1 / 2$, in turn, destroys the balance producing a zero mode in the electromagnetic case. There is no zero mode in the gravitomagnetic field and, as a result, the axial anomaly in gravitational field is proportional to the curvature rather than connection.

Note that the fundamental $U(1)$ chiral anomaly is manifested phenomenologically in two different types of instability of chiral media. First, in presence of external electric, $\boldsymbol{E} \neq 0$ and magnetic, $\boldsymbol{B} \neq 0$, fields such that $\int d^{3} x(\boldsymbol{E} \cdot \boldsymbol{B}) \neq 0$ chiral imbalance of fermions is developed. In other words, there is transition

$$
\text { (helical magnetic field }) \rightarrow(\text { chiral imbalance }) .
$$

However, the inverse process

$$
\text { (chiral imbalance) } \rightarrow \text { (helical magnetic field), }
$$

is also possible, for recent analysis and further references see, e.g., [38]. In the gravitational case, we discussed so far production of chiral imbalance stimulated by an external gravitational field. According to [35] the Hawking radiation of chiral fermions can be considered as a manifestation of the gravitational chiral anomaly. A reversed process, that is a decay of the chiral imbalance into a gravitational field would be most amusing to observe. In perspective, such studies might be made possible by progress in lattice simulations.

\section{CONCLUSIONS}

Basing on the ansatz for the Wigner function proposed in [16], we obtained simple formulas for the axial current in the general case of massive fermions, see Eqs. (2.5) and (2.6). In these formulas, the angular velocity and acceleration enter the Fermi-Dirac distribution in combination with the chemical potential. The zero-mass limit (2.8), (2.10), which is consistent in the linear approximation with the standard formula for the CVE, was studied. It is shown that in this case the axial current has a series of discontinuities and cusps at a temperature lower than the Unruh temperature, which look like Roberge-Weiss phase transitions in theories with imaginary chemical potential. In more general case of an arbitrary mutual orientation of the acceleration and angular velocity, the temperature (2.9) appears as a boundary, instead of the Unruh temperature and the boundary of allowed values of acceleration and temperature shifts as is shown in Fig. 1 on the left, which can be considered as a possible generalisation of the prediction for this boundary in [17] for accelerated medium without rotation.

Dependence of the axial current on the mass of constituents implied by Eq. (2.5) was investigated. In the limit $T=a_{\mu}=0,(2.5)$ reduces to (3.1), and the axial current, as a function of the angular velocity and chemical potential, vanishes in the two-dimensional region $\Omega<2(m-|\mu|)$, as is shown in Fig. 1 on the right.

The obtained results are based on the assumption that the fermion interaction is weak, but, given the connection between the effects studied and the quantum anomalies and also the Unruh effect, as well as the fact of conservation of the chiral effects in the hydrodynamic approximation, we can assume that at least in part they can be transferred to strong-coupling case.

One can see, that the Wigner-function approach in the zero-mass limit reproduces, after the integration over momenta, the anomaly induced contribution to the axial current, establishing the relation between different approaches to polarization. One can even say, that the thermodynamical approach contains the "hidden anomaly."

\section{ACKNOWLEDGMENTS}

Useful discussions with F. Becattini, W. Florkowski, E. Grossi, A. Sorin, E. Speranza and A. Starobinsky are gratefully acknowledged. The work was supported by Russian Science Foundation Grant No. 16-12-10059.
[1] A. Vilenkin, Macroscopic parity violating effects: Neutrino fluxes from rotating black holes and in rotating thermal radiation, Phys. Rev. D 20, 1807 (1979).

[2] A. Vilenkin, Quantum field theory at finite temperature in a rotating system, Phys. Rev. D 21, 2260 (1980).

[3] A. Vilenkin, Equilibrium parity violating current in a magnetic field, Phys. Rev. D 22, 3080 (1980).
[4] D. E. Kharzeev, K. Landsteiner, A. Schmitt, and H. U. Yee, 'Strongly interacting matter in magnetic fields': An overview, Lect. Notes Phys. 871, 1 (2013).

[5] K. Fukushima, D. E. Kharzeev, and H. J. Warringa, The chiral magnetic effect, Phys. Rev. D 78, 074033 (2008).

[6] D. T. Son and P. Surowka, Hydrodynamics with Triangle Anomalies, Phys. Rev. Lett. 103, 191601 (2009). 
[7] S. Golkar and D. T. Son, (Non)-renormalization of the chiral vortical effect coefficient, J. High Energy Phys. 02 (2015) 169.

[8] Y. Neiman and Y. Oz, Relativistic hydrodynamics with general anomalous charges, J. High Energy Phys. 03 (2011) 023.

[9] A. V. Sadofyev, V. I. Shevchenko, and V. I. Zakharov, Notes on chiral hydrodynamics within effective theory approach, Phys. Rev. D 83, 105025 (2011).

[10] V. I. Zakharov, Chiral magnetic effect in hydrodynamic approximation, Lect. Notes Phys. 871, 295 (2013).

[11] M. Buzzegoli, E. Grossi, and F. Becattini, General equilibrium second-order hydrodynamic coefficients for free quantum fields, J. High Energy Phys. 10 (2017) 091.

[12] J. H. Gao, Z. T. Liang, S. Pu, Q. Wang, and X. N. Wang, Chiral Anomaly and Local Polarization Effect from Quantum Kinetic Approach, Phys. Rev. Lett. 109, 232301 (2012).

[13] J.h. Gao, S. Pu, and Q. Wang, Covariant chiral kinetic equation in the Wigner function approach, Phys. Rev. D 96, 016002 (2017).

[14] K. Landsteiner, E. Megias, and F. Pena-Benitez, Anomalous transport from Kubo formulae, Lect. Notes Phys. 871, 433 (2013).

[15] G. Prokhorov and O. Teryaev, Anomalous current from the covariant Wigner function, Phys. Rev. D 97, 076013 (2018).

[16] F. Becattini, V. Chandra, L. Del Zanna, and E. Grossi, Relativistic distribution function for particles with spin at local thermodynamical equilibrium, Ann. Phys. (Amsterdam) 338, 32 (2013).

[17] F. Becattini, Thermodynamic equilibrium with acceleration and the Unruh effect, Phys. Rev. D 97, 085013 (2018).

[18] W. Florkowski, E. Speranza, and F. Becattini, Perfect-fluid hydrodynamics with constant acceleration along the stream lines and spin polarization, Acta Phys. Pol. B 49, 1409 (2018).

[19] M. Baznat, K. Gudima, A. Sorin, and O. Teryaev, Hyperon polarization in heavy-ion collisions and gravity-related anomaly, Phys. Rev. C 97, 041902 (2018).

[20] O. Rogachevsky, A. Sorin, and O. Teryaev, Chiral vortaic effect and neutron asymmetries in heavy-ion collisions, Phys. Rev. C 82 (2010) 054910.

[21] A. Sorin and O. Teryaev, Axial anomaly and energy dependence of hyperon polarization in heavy-ion collisions, Phys. Rev. C 95, 011902 (2017).

[22] M. Baznat, K. Gudima, A. Sorin, and O. Teryaev, Helicity separation in heavy-ion collisions, Phys. Rev. C 88, 061901 (2013); Erratum, Phys. Rev. C 93, 031902(E) (2016);
Hyperons polarization in heavy-ion collisions, EPJ Web Conf. 138, 01008 (2017).

[23] F. Becattini and I. Karpenko, Collective Longitudinal Polarization in Relativistic Heavy-Ion Collisions at Very High Energy, Phys. Rev. Lett. 120, 012302 (2018).

[24] F. Becattini, I. Karpenko, M. Lisa, I. Upsal, and S. Voloshin, Global hyperon polarization at local thermodynamic equilibrium with vorticity, magnetic field and feed-down, Phys. Rev. C 95, 054902 (2017).

[25] I. Karpenko and F. Becattini, Vorticity in the QGP liquid and $\Lambda$ polarization at the RHIC Beam Energy Scan, Nucl. Phys. A967, 764 (2017).

[26] S. R. De Groot, W. A. Van Leeuwen, and C. G. Van Weert, Relativistic Kinetic Theory: Principles and Applications (North-Holland, Amsterdam, 1980), p. 417.

[27] W. Florkowski, B. Friman, A. Jaiswal, and E. Speranza, Relativistic fluid dynamics with spin, Phys. Rev. C 97, 041901 (2018).

[28] A. Roberge and N. Weiss, Gauge theories with imaginary chemical potential and the phases of QCD, Nucl. Phys. B275, 734 (1986).

[29] Y. Sakai, K. Kashiwa, H. Kouno, and M. Yahiro, Phase diagram in the imaginary chemical potential region and extended Z(3) symmetry, Phys. Rev. D 78, 036001 (2008).

[30] A. P. Prudnikov, Yu. A. Brychkov, and O. I. Marichev, Integrals and Series, More Special Functions Vol. 3 (Gordon \& Breach Science Publishers, New York, 1990).

[31] V.E. Ambru and E. Winstanley, Rotating quantum states, Phys. Lett. B 734, 296 (2014).

[32] P. Ramond, Field theory: A modern primer, Front. Phys. 51, 1 (1981); Erratum, Front. Phys. 74, 1(E) (1989).

[33] M. Stone and J. Kim, Mixed anomalies: Chiral vortical effect and the Sommerfeld expansion, Phys. Rev. D 98, 025012 (2018).

[34] V. I. Zakharov, Notes on conservation laws in chiral hydrodynamics, arXiv:1611.09113.

[35] S. P. Robinson and F. Wilczek, A Relationship Between Hawking Radiation and Gravitational Anomalies, Phys. Rev. Lett. 95, 011303 (2005); S. Iso, H. Umetsu, and F. Wilczek, Anomalies, Hawking radiations and regularity in rotating black holes, Phys. Rev. D 74, 044017 (2006).

[36] L. Alvarez-Gaume and E. Witten, Gravitational anomalies, Nucl. Phys. B234, 269 (1984).

[37] O. V. Teryaev, Gravitational form factors and nucleon spin structure, Front. Phys. 11, 111207 (2016).

[38] K. Tuchin, Taming instability of magnetic field in chiral medium, Nucl. Phys. A969, 1 (2018). 